\title{
Hyperlipidemia of Pregnancy: Normal or Predictor of Preeclampsia
}

\author{
Suman Chaudhary ${ }^{1}$, Manju Hiranwal ${ }^{2}$, Deepa Chaudhary ${ }^{3}$, Pradeep Dudi ${ }^{4}$
}

\begin{abstract}
Aim: Pregnant women develop physiological dyslipidemia that can be measured in the laboratory by the lipid indexes. The atherogenic lipid profile of human gestation is further enhanced in preeclampsia. Our study has two purposes. First, the study of the alterations of total cholesterol $(\mathrm{TCH})$, triglycerides (TG), low-density lipoprotein (LDL), very-low-density lipoprotein (VLDL), and high-density lipoprotein (HDL) during the pregnancy. The values were compared between early and late pregnancy. Second, the values of women who remained normotensive throughout pregnancy were compared to those who developed hypertension later on.

Materials and methods: A total of 500 pregnant women with gestational age of 20 weeks. All of them were followed till delivery and maternal and fetal outcome was noted.

Results: Out of 500 pregnant women, 45 were lost to follow-up. The mean age of study population was $23.27 \pm 3.54$ years. A total of 47 (10.33\%) developed preeclampsia-22 had severe and 25 had mild hypertension. All plasma lipid levels increase as gestational age progresses except HDL. This change is statistically significant. It is observed that pregnant women with high TCH and LDL levels at $<20$ weeks are more likely to develop hypertension later ( $p$ value $<0.05$ ). Hypertensive pregnant women has very high TCH and LDL levels at $>37$ weeks $(p$ value $<0.05)$ as compared to normotensive pregnant women.

Conclusion: Pregnancy, in general preeclamptic pregnancy, in particular is associated with marked hyperlipidemia. The study suggests that alterations in lipid metabolism observed in preeclampsia are present at early gestational age $<20$ weeks. Any pregnant women with higher plasma lipid levels may be observed and evaluated for preeclampsia.

Keywords: High-density lipoprotein, Lipid profile, Low-density lipoprotein, Preeclampsia, Pregnancy, Total cholesterol, Triglycerides.

Journal of South Asian Federation of Obstetrics and Gynaecology (2020): 10.5005/jp-journals-10006-1752
\end{abstract}

\section{INTRODUCTION}

Profound local and systemic changes in maternal physiology are initiated by conception and continued throughout pregnancy. ${ }^{1}$ During early pregnancy, the maternal metabolic environment is modified by a rise in serum levels of estrogen and progesterone, pancreatic $\beta$-cell hyperplasia occurs, and there is an increase in the secretion of insulin. ${ }^{2}$

As pregnancy advances, a well-integrated and systematic metabolic shift occurs to provide sufficient and balanced supply of nutrients to a constantly feeding fetus from an intermittently fasting and feeding mother. Freinkel ${ }^{3}$ was the first to describe the maternal metabolic changes of late pregnancy as "accelerated starvation," when food is unavailable, and "facilitated anabolism," when food is ingested. Chauffard ${ }^{4}$ in 1911 undertook the first chemical study of blood lipids during pregnancy and suggested that an increase occurs in the cholesterol level. Later on, multiple studies demonstrated increase in various fractions of lipids and lipid indices such as total cholesterol (TCH), triglycerides (TG), highdensity lipoprotein (HDL), low-density lipoprotein (LDL) and verylow-density lipoprotein (VLDL). That is, pregnant women develop physiological hyperlipidemia. $^{5}$

The alterations of serum lipid indices are associated with the gestational age. The increase of the lipid and lipoprotein metabolism reaches the level of cardiovascular risk ${ }^{6,7}$ during the second trimester. In the later half of pregnancy, there is two- to threefold increase in plasma triglycerides and lesser increase in $\mathrm{TCH}, \mathrm{HDL}-\mathrm{C}$, and LDL-C.

The hyperlipidemia of the second half of pregnancy may be a purely physiological response or may be indicative of pathology

\footnotetext{
${ }^{1-3}$ Department of Obstetrics and Gynecology, SMS Medical College, Jaipur, Rajasthan, India

${ }^{4}$ Department of Radio Diagnosis, Railway Hospital, Jaipur, Rajasthan,
} India

Corresponding Author: Suman Chaudhary, Department of Obstetrics and Gynecology, SMS Medical College, Jaipur, Rajasthan, India, Phone: +919460077909, e-mail: drsuman1981@gmail.com

How to cite this article: Chaudhary S, Hiranwal M, Chaudhary D, et al. Hyperlipidemia of Pregnancy: Normal or Predictor of Preeclampsia. J South Asian Feder Obst Gynae 2020;12(1):31-33.

Source of support: Nil

Conflict of interest: None

in some women. In the present times, where prevalence of cardiovascular diseases is on rise, we must evaluate whether the hyperlipidemic response to pregnancy is a variable or not and if so, whether it can predict future hyperlipidemia in a manner analogous to that of impaired glucose tolerance during pregnancy, which can predict DM in later life of women. There is evidence that triglyceride concentration, if measured between 9 weeks and 12 weeks of gestation, has moderate predictive value for subsequent glucose tolerance in pregnancy. For that reason, further studies are needed to investigate the role of early measurement in the screening and management of cardiovascular damage. , $^{8}$

Late pregnancy is associated with the formation of susceptible oxidizable particles and an increase in oxidative damage. These biochemical changes may be relevant for the long-term cardiovascular health of women, especially those of high parity who are at high risk for cardiovascular disease (e.g., women with diabetes)., ${ }^{9,10}$ 
Preeclampsia accentuates atherogenic lipid profile changes associated with human gestation, which may be a potential contributor to endothelial cell dysfunction. Studies have shown $n^{9,11}$ that in preeclampsia, the plasma lipids profile is substantially above levels seen in normal pregnancies. Such lipid changes may play a role in the endothelial damage characteristic of preeclampsia.

Our study aimed to address two objectives:

First, the study of the alterations of TCH, TG, LDL,VLDL, and $\mathrm{HDL}$ during the pregnancy, i.e., at $<20$ weeks and at $>37$ weeks. The values were compared between early and late pregnancy and analyzed.

Second, the values of women who remained normotensive throughout pregnancy were compared to those who developed hypertension later on at both early and late pregnancy.

\section{Materials and Methods}

A total of 500 pregnant women with gestational age $<20$ weeks were enrolled for study after informed consent. Fasting venous blood samples were collected into plain tubes and subjected to estimation of the lipid profile by following methods: serum cholesterol by the modified Roeschlau's method, serum triglyceride by the McGrowan method, HDL-C by the Bursteim et al. method, and VLDL and LDL-C was calculated by Friedewald's formula. Estimation of the lipid profile was repeated at gestational age $>37$ weeks. All of them were followed till delivery and the maternal and fetal outcome was noted.

\section{Results}

Out of 500 pregnant women, 45 were lost to follow-up.

- The mean age of study population was $23.27 \pm 3.54$. A total of $233(51.21 \%)$ of study population were of age $21-25$ years.

- A total of 242 (53.19\%) of women were multigravidas and 213 (46.81\%) were primigravidas.

- Out of 455 women, 47 (10.33\%) developed gestational hypertension -22 had severe hypertension and 25 had mild hypertension.

- Out of 455 women, 23 (5.06\%) had IUGR fetus.

- All lipid lipoprotein levels increase as gestational age progresses except HDL. This change is statistically significant too (Tables 1 and 2).

- Comparison of the plasma lipid profile at $<20$ weeks in normotensive pregnant women and pregnant women developing hypertension shows that pregnant women who developed hypertension later on had higher levels of TG, TCH, LDL, VLDL, and low levels of HDL (Table 3).

- It is clear that pregnant women with high TCH and LDL levels at 20 weeks are more likely to develop hypertension later ( $p$ value $<0.05$ )

Table 1: Distribution of cases according to parity and age

\begin{tabular}{lccc}
\hline Age/parity & Primigravida & Multigravida & Total \\
\hline$<20$ years & 83 & 6 & 89 \\
21-25 years & 68 & 165 & 233 \\
26-30 years & 52 & 64 & 116 \\
$>30$ years & 10 & 7 & 17 \\
Total & 213 & 242 & 455 \\
\hline
\end{tabular}

The mean age of study population was $23.27 \pm 3.54$ years
Table 2: Variation in plasma lipid profile during pregnancy

\begin{tabular}{lccl}
\hline Parameters $(\mathrm{mg} / \mathrm{dL})$ & $<20$ weeks & $>37$ weeks & p value \\
\hline TG & $162.58 \pm 68.31$ & $179.41 \pm 46.31$ & 0.0001 \\
TCH & $187.40 \pm 36.87$ & $201.26 \pm 48.57$ & 0.0001 \\
HDL & $40.11 \pm 7.74$ & $36.70 \pm 5.89$ & 0.0001 \\
LDL & $115.83 \pm 28.0 .36$ & $120.89 \pm 26.97$ & 0.005 \\
VLDL & $26.24 \pm 29.29$ & $29.91 \pm 6.77$ & 0.009 \\
\hline
\end{tabular}

All lipid lipoprotein levels increase as gestational age progresses except $\mathrm{HDL}$. This change is statistically significant too

Table 3: Comparison of plasma lipid profile at $<20$ weeks in normotensive pregnant women and pregnant women developing hypertension

\begin{tabular}{lccl}
\hline Parameters $(\mathrm{mg} / \mathrm{dL})$ & Normotensive & Hypertensive & p value \\
\hline TG & $168.77 \pm 31.66$ & $175.00 \pm 39.90$ & 0.188 \\
$\mathrm{TCH}$ & $182.95 \pm 38.79$ & $200.82 \pm 40.15$ & 0.008 \\
$\mathrm{HDL}$ & $43.50 \pm 13.78$ & $39.00 \pm 4.76$ & 0.297 \\
$\mathrm{LDL}$ & $103.022 \pm 3.85$ & $125.82 \pm 31.75$ & 0.015 \\
VLDL & $21.25 \pm 6.10$ & $28.36 \pm 7.01$ & 0.602 \\
\hline
\end{tabular}

It is clear that pregnant women with high TCH and LDL levels at 20 weeks are more likely to develop hypertension later $(p$ value $<0.05$ )

Table 4: Comparison of plasma lipid profile at > 37 weeks in normotensive pregnant women and pregnant women developing hypertension

\begin{tabular}{lccl}
\hline Parameters $(\mathrm{mg} / \mathrm{dL})$ & Normotensive & Hypertensive & pvalue \\
\hline TG & $184.74 \pm 49.80$ & $194.95 \pm 50.52$ & 0.188 \\
TCH & $213.01 \pm 57.23$ & $302.38 \pm 52.60$ & 0.0001 \\
HDL & $38.14 \pm 6.22$ & $37.76 \pm 5.53$ & 0.297 \\
LDL & $110.80 \pm 19.92$ & $127.44 \pm 33.51$ & 0.010 \\
VLDL & $30.19 \pm 7.18$ & $31.70 \pm 8.79$ & 0.602
\end{tabular}

Pregnant women with hypertension have very high TCH and LDL levels at $>37$ weeks ( $p$ value $<0.05$ ) as compared to normotensive pregnant women

- Table 4 shows comparison of the plasma lipid profile at $>37$ weeks in normotensive pregnant women and pregnant women with hypertension. All lipid levels are highly elevated in women with hypertension.

- Pregnant women with hypertension have very high TCH and LDL levels at $>37$ weeks ( $p$ value $<0.05$ ) as compared to normotensive pregnant women.

\section{Discussion}

Age is one of the important factors that affects the serum lipid profile. In the present study, the mean age of study population was $23.27 \pm 3.54$ years. A total of $233(51.21 \%)$ of study population were of age 21-25 years; thus, most of them were young.

In our study, we found that all lipid indices increase with increasing gestational age; however, a drop in HDL was observed. This change is highly significant ( $p$ value $<0.05$ ).

This finding is in congruence with studies done by Parchwani et al., ${ }^{12}$ Kumar et al., ${ }^{13}$ and Blessings et al. ${ }^{9}$ Similar to our study results, several other studies conducted by Fahraeus et al., ${ }^{14}$ Jimenez et al., ${ }^{15}$ and Potter and Neste ${ }^{16}$ reported similar observations. The principal modulator of this hypertriglyceridemia is estrogen as pregnancy is associated with hyperoestrogenemia. Estrogen induces hepatic 
biosynthesis of endogenous triglycerides, which is carried by VLDL. ${ }^{17}$ This process may be modulated by hyperinsulinemia found in pregnancy. ${ }^{18}$

We observed that pregnant women with high TCH ( $p$ value 0.008 ) and high LDL ( $p$ value 0.015 ) at gestational age $<20$ weeks were more likely to develop preeclampsia as compared to women with normotensive pregnancy. This observation suggests a positive association between pregnancy-induced hyperlipidemia and subsequent risk of preeclampsia. Similar results were observed in studies by Gratacos et al., ${ }^{19}$ Deshpande et al., ${ }^{20}$ Singh et al. ${ }^{21}$

We also observed that pregnant women with preeclampsia had high plasma lipid levels, especially TCH ( $p$ value $<0.0001)$ and LDL ( $p$ value $<0.001$ ), at gestational age $>37$ weeks as compared to normotensive pregnant women. This observation supports role of pregnancy-induced hyperlipidemia in preeclampsia.

\section{Conclusion}

The study results suggest that pregnancy-induced hyperlipidemia observed in preeclampsia is present at early gestational age $(<20$ weeks). Our results suggested that maternal hyperlipidemia was significantly associated with risk of developing preeclampsia. Any pregnant women detected with higher serum plasma lipid levels in early gestation may be observed and evaluated for preeclampsia.

It may also have long-term effect on health of women and lead to significant risk of development of obesity, atherosclerosis, type 2 diabetes mellitus, etc. Therefore, it is advisable to screen pregnant women for the serum lipid profile.

\section{References}

1. Kortenoever M. Physiological changes in pregnancy. Int J Contemp Med Res 1960;21:443-445.

2. Kalkhoff RK. Metabolic effects of progesterone. Am J Obst Gynae 1982;142(6):735-737. DOI: 10.1016/S0002-9378(16)32480-2.

3. Freinkel N. Effect of the conceptus on maternal metabolism during pregnancy. Excerpta Med 1964;12:679-681.

4. Chauffard A, Laroche G, Grigaut A. Blood lipids in pregnancy. Obstetriqie 1911;4:481-482.

5. Merabishvili V, Kambladze O, Sulaberidze T. Peculiarities of lipid metabolism during pregnancy. Georgian Med News 2006;138:86-89.

6. Lippi G, Albiero A, Montagnana M, et al. Lipid and lipoprotein profile in physiological pregnancy. Clin Lab 2007;53(3-4):173-177.
7. Loke DF, Viegas $O A$, Kek $L P$, et al. Lipid profiles during and after pregnancy. Gynecol Obstet Invest 1991;32(3):144-147. DOI: 10.1159/000293016.

8. Brizzi P, Tonolo G, Esposito F, et al. Lipoprotein metabolism during normal pregnancy. Am J Obstet Gynecol 1999;181(2):430-434. DOI: 10.1016/s0002-9378(99)70574-0.

9. Okojie FO, Blessing IO, Mable AE, et al. Comparative study of lipid profile of normal pregnant women in the different trimesters. Arch Appl Sci Red 2011;3(3):528-532.

10. Martin U, Davies $C$, Hayavi S, et al. Is normal pregnancy atherogenic? Clin Sci (Lond) 1999;96(4):421-425. DOI: 10.1042/cs0960421.

11. Bodnar M, Ness B, Hanger F, et al. Inflammation and triglycerides partially mediate the effect of pre-pregnancy body mass index on the risk of preeclampsia. Am J Epidemiol 2005;162(12):1198-1206. DOI: 10.1093/aje/kwi334.

12. Parchwani $D$, Patel $D$. Status of lipid profile in pregnancy. Nat J Med Res 2011;1(1):10-12.

13. Kumar S, Dadheech G, Gupta RC. Dyslipidemia in pregnancy in a rural population in North India. Int Res J Biochem Biotech 2014;1(1): 002-004.

14. Fahraeus L, Larsson-Cohn U, Wallentin L. Plasma lipoproteins including high density lipoprotein subfractions during normal pregnancy. Obstet Gynecol 1995;66:468-472.

15. Jimenez DM, Pocovi M, Ramon C. Longitudinal study of plasma lipids and lipoprotein cholesterol in normal pregnancy and puerpurium. J Gynecol Obstet Invest 1988;25(3):158-164. DOI: 10.1159/000293765.

16. Potter JM, Neste PJ. The hyperlipidemia of pregnancy in normal and complicated pregnancies. Am J Obstet Gynecol 1979;133(2):165-179. DOI: 10.1016/0002-9378(79)90469-1.

17. Glueck CJ, Fallet RW, Scheel D. Effects of oestrogenic compounds on triglycerides kinetics. Metabolism 1975;24(4):537-545. DOI: 10.1016/0026-0495(75)90078-5.

18. Adegke $\mathrm{OA}$, lyare $\mathrm{EE}$, Gbenebitse SO. Fasting plasma glucose and cholesterol levels in pregnant Nigerian women. Niger Postgrad Med J 2003;10(1):32-36.

19. Gratacos E, Casals E, Gomez O, et al. Increased susceptibility to low density lipoprotein oxidation in women with a history of preeclampsia. BJOG 2003;110(4):400-404. DOI: 10.1046/j.14710528.2003.02349.x.

20. Deshpande H, Chandtakant M, Poonam V, et al. Study of serum lipid profile in pregnancy induced Hypertension. Indian J Appl Res 2016;6:546-548.

21. Singh DU, Yadav $S$, Mehrotra $S$, et al. Serum lipid profile in early pregnancy as a predictor of preeclampsia. Int J Med Res Rev 2013;1(02). DOI: 10.17511/ijmrr.2013.i02.03. 